treatment on the physical properties of metals. The phase rule, which is briefly explained, could be given a practical application by referring to the nature of, alloys, particularly in the case of carbon-iron mixtures.

As is the case with all the works published by the well known firm of Ulrico Hoepli, the printing and reproduction of the illustrations leave nothing to be desired. It is, however, a pity that so many proper names are wrongly spelt; thus Graham is uniformly spelt Grahm, and Van der Waals Van der Vaals. More than ten misprints of other names are observable.

W. A. D.

\section{A NEW CRYSTALLOGRAPHY.}

Grundzüge der Kristallographie. By Prof. C. M. Viola. Pp. iv +389 . (Leipzig: W. Engelmann, I904.) Price II marks; bound, I2 marks.

THE opinion is rapidly gaining ground that the theory of crystallography based on the laws of rational indices and symmetry no longer suffices without modification for the classification and description of crystals. It is recognised on the one hand that isomorphism of kindred substances shows itself (as in the Humite group of minerals) more in similarity of crystalline habit and angles than in identity of optical and geometrical symmetry, and on the other hand that vicinal faces with high indices may play an important part in the economy of crystals. Prof. Viola is evidently of opinion that the old methods cannot be adapted to meet the situation, and his book is as revolutionary as it well could be. Crystals are here divided into 7 sygonies, Io fundamental forms, and 29 harmonies; symmetry is but a particular case of harmony; twins are two similar crystals with two predominant elements in common; the number of space-lattices is reduced to Io, and of space-groups to ${ }_{15} 6$. The basis of classification is descriptive, not geometrical; blende, felspar, and garnet belong to the same fundamental form, chalcopyrite and tetrahedrite to the same harmony.

If the author had merely attacked the existing theory and advocated a classification expressing the results of direct observation alone, independent of any hypothesis, he might have had some success. Unfortunately, he has tried to build up a mathematical theory of his own, with disastrous results. The average shape of all crystals of a substance grown under approximately the same conditions is its "habit"; the average shape of all habits is its " fundamental form." The rate of growth in any direction is proportional to the " cohesion" in that direction (measured, apparently, by the force needed to break a rod of the substance the length of which lies in the given direction), and cleavage takes place perpendicular to the lines in which minima of cohesion are well marked. It follows that the fundamental form has always a centre of symmetry. These assumptions are hardly justified by the cleavage and usual habit of many crystals, e.g. fluorite and tetrahedrite, but the mathematical development of these hypotheses is, if possible, still more unfortunate than the premises themselves. It is argued (p. I4, cf. Fig. 20) that if two faces grow outwards with velocities $c_{1}$ and $c_{2}$, (I) their intersection moves with the velocity $c_{3}$, compounded of $c_{1}$ and $c_{2}$, (2) therefore the face perpendicular to $c_{3}$ grows with velocity $c_{3},(3) c_{3}$ is a maximum or a minimum when $c_{2}$ and $c_{2}$ are minima. Of these statements (I) and (3) are untrue, and (2) absolutely unproven. Thus the fundamental principles on which nearly the whole of the book is based are wrong. Much of the reasoning is of the same fallacious nature, or is, at best, only an appeal to probability; but one more example must suffice.

The author sets himself (p. 25r) the impossible task of proving that a symmetry-axis of a homogeneous medium is 2 -al, 3 -al, 4 -al, or 6 -al without employing either the law of rational indices or a molecular structure. He accomplishes this by assuming that if the medium is brought to self-coincidence by a rotation through an angle $2 \gamma$ about an axis $\mathrm{C}$, it cannot be brought to self-coincidence by a rotation about $\mathrm{C}$ through any angle less than $2 \gamma$.

Prof. Viola apparently considers the space-lattice as only a convenient geometrical expression of the physical properties of a crystal, not as corresponding to any reality of crystal-structure. It is true that he proves (by assuming that the densities of the molecule and of the crystal as a whole are equal, see pp. 280 , 335) that the unit of crystalline structure must be the same as the chemical molecule; but on pp. $3^{22}$ and 334 he uses arguments which would prove the existence of an infinite number of such units in a finite volume.

Crystallographers owe a debt of gratitude to the author for his clear and complete lists of references to the literature of the various subjects with which he deals; the historical notes are also very valuable. The chapters on the two-circle goniometer and the stereographic projection contain much that is interesting and not in the usual text-books. The appearance of the book is attractive, but there is a large number of misprints, some of which quite obscure the author's meaning.

Harold Hilton.

\section{OUR BOOK SHELF.}

The Arris and Gale Lectures on the Neurology of Vision. By J. Herbert Parsons, B.S., D.Sc., F.R.C.S. Pp. 7o. (London: Hodder and Stoughton, r904.) Price $2 s .6 d$. net.

The two lectures delivered by Mr. Parsons in the spring of last year before the Royal College of Surgeons deal with some points on the neurology of the eye which are of extreme interest. The first lecture has for its subject the course of the afferent impulses from the retina to the central nervous system, and their final distribution in the cerebral cortex. Since the delivery of these lectures there have been several important contributions to this latter subject. The case of Dr. Beevor and Dr. Collier, reported in the summer number of Brain, seems to go conclusively against the more restricted visual area for which Henschen argues. In this case, despite the fact that the lingual lobe, the depths of the calcarine fissure, and the lower cuneal lobe were all affected, the restriction of the field of vision was simply quadrantic. The truth seems to be that the limits of the visual cortical area correspond to the limits of the layer of Gennari, and that this varies markedly in its relations to the surface in different cases.

The second lecture deals with an equally important NO. I $84 \mathrm{I}$, VOL. $7 \mathrm{I}]$ 